\title{
Design, Validity and Reliability of the Cancer-Related Fatigue Questionnaire in Children with Cancer Aged 4 - 18 Years
}

\author{
Mahdieh Momayyezi ${ }^{1}$, Hossein Fallahzadeh ${ }^{1,{ }^{*}}$, Fatemeh Farzaneh ${ }^{2}$, Mohammad Momayyezi ${ }^{3}$ and \\ Soheila Bazm ${ }^{4}$ \\ ${ }^{1}$ Research Center of Prevention and Epidemiology of Non-Communicable Disease, School of Public Health, Shahid Sadoughi University of Medical Sciences, Yazd, Iran \\ ${ }^{2}$ Shahid Sadoughi University of Medical Sciences,Yazd, Iran \\ ${ }^{3}$ School of Medicine, Shahid Sadoughi University of Medical Sciences, Yazd, Iran \\ ${ }^{4}$ School of Public Health, Shahid Sadoughi University of Medical Sciences, Yazd, Iran \\ "Corresponding author: Research Center of Prevention and Epidemiology of Non-Communicable Disease, Shahid Sadoughi University of Medical Sciences, Yazd, Iran. Tel: \\ +98-9131529486, Fax: +98-3538209119, Email: hofaab@yahoo.com
}

Received 2018 January 16; Revised 2018 June 23; Accepted 2018 July 12.

\begin{abstract}
Background: Children and adolescents with cancer experience some complications such as pain, depression, and fatigue that impact the quality of life. Fatigue is one of the most common cancer treatment problems in most children with cancer.

Objectives: The purpose of this study was to construct a comprehensive instrument to examine fatigue in patients with cancer (4 18 years).

Methods: This descriptive study was conducted on 101 children with cancer. A questionnaire was designed to examine fatigue in patients with cancer ( 4 - 18 years) using similar studies. The questionnaire reliability was determined using the Cronbach's alpha and test-retest method. Face validity of the questionnaire was assessed by professionals. To determine the construct validity, factor analysis was run. Data were analyzed using SPSS (version 16).

Results: The mean age of the patients was $9.54 \pm 3.97$ years and the mean duration of the cancer $12.82 \pm 2.66$ months. Cronbach's alpha for whole scale and for all dimensions was 0.93 and more than 0.8 (excellent score). Intraclass correlation coefficient (ICC) for whole scale and for all dimensions of questionnaire was 0.92 and more than 0.8 , respectively. According to factor analysis method, three extracted dimensions of the questionnaire were: daily activities and general problems (11 items), sleep problems ( 9 items), as well as mental states and emotions (5 items).

Conclusions: This study showed that validity and reliability of the cancer-related fatigue questionnaire for patients with cancer (4 -18 years) are acceptable and suitable for Iranian culture.
\end{abstract}

Keywords: Child, Epidemiologic Study, Fatigue, Reproducibility of Results, Sensitivity and Specificity, Logic

\section{Background}

Despite efforts to control communicable diseases in childhood, the rise of non-communicable diseases like cancer is increased (1). Although cancer is rare among children under 15 years old, leukemia, central nervous system tumors (CNS), and lymphoma are the most common childhood cancers (2).

According to World Health Organization's estimation, the incidence rate of childhood cancer is 100 in 1000000 children (3). This rate in Iranian children is 48 - 112 per 1000 000 and 51-144 per 1000000 for boys and girls in different geographical areas in Iran, respectively (2). Cancer is the second common cause of death among children in developed countries (4). A study conducted by American Cancer Society reported that the five year survival rate of Pediatric cancer is approximately $80 \%$ at the present time (5).

Cancer patients experience some degree of pain, depression and fatigue, which can have a negative effect on quality of life (6). Fatigue is seen as one of the most distressing complaints of cancer treatment in $80 \%$ of children with cancer (7).

Cancer-related fatigue (CRF) is a subjective experience (8). It is identified as the most prevalent symptom of cancer that significantly interferes with quality of life (9) and usually increases during treatment (10). CRF can be effective on ability to conduct daily activities and roles as well as lead to delays in treatment and may continue in patients after recovery (11). The National Comprehensive Cancer Network (NCCN) defined CRF as "a distressing, persistent, subjective sense of physical, emotional and/or cognitive

Copyright (C) 2018, Journal of Comprehensive Pediatrics. This is an open-access article distributed under the terms of the Creative Commons Attribution-NonCommercial 4.0 International License (http://creativecommons.org/licenses/by-nc/4.0/) which permits copy and redistribute the material just in noncommercial usages, provided the original work is properly cited. 
tiredness or exhaustion related to cancer or cancer treatment that is not proportional to recent activity and interferes with usual functioning" (12).

A survey on parents of children with cancer showed that $49 \%$ of parents reported the symptom of fatigue in their children (13). Another study also showed $86 \%$ of parents reported that their children had the symptom of physical fatigue (14). The etiology of cancer-related fatigue is still not completely understood. Fatigue in patients with cancer is a multifactorial problem. It may be due to biological mechanisms and psychological/behavioral effects such as stress and depression (15). Some studies have shown that carnitine deficiency in cancer patients (due to decreased intake and metabolic needs, also treatment with medications that interfere with carnitine metabolism (16)) may promote the risk of the development of chronic fatigue (17). There are no adequate studies in Iran to assess fatigue in children with cancer. Therefore, researchers need to have a valid and reliable instrument for the assessment of fatigue for increasing the number of studies in Iran. Moreover, this study aimed to introduce a comprehensive instrument to examine the fatigue in patients with cancer (4 -18 years).

\section{Methods}

\subsection{Study Setting and Sample}

This descriptive study was conducted on 101 children with cancer. The samples of this study were children and adolescents aged 4 - 18 years who are suffering from cancer and referred to Shahid Sadoughi Hospital in Yazd. The sample size was determined to be 97 based on a confidence interval of $95 \%$ and standard deviation of 3.5 based on similar studies (18) and error estimation of mean of 0.7, which was increased to 101 to account for potential missing cases. Inclusion criteria were definitive diagnosis of cancer, begin the treatment process, less than 18 years of age, and satisfaction to participate.

Researchers met with participants at Shahid Sadoughi Hospital in Yazd. Researchers explained the aim of the study to participants; then, completed the questionnaire by face-to-face interview with parents of children with cancer.

\subsection{Instrumentation}

In this study, data were collected by questionnaire, which were filled out with the caregivers. The first part of the questionnaire, which was designed to examine cancer-related fatigue, included demographic variables and disease-related ones. Initially, to design the questionnaire the researchers reviewed some studies and questionnaires related to fatigue such as multidimensional fatigue inventory (MFI) and fatigue severity scale (FSS) in patients suffering from cancer. Then, factors affecting cancerrelated fatigue were collected. Finally, a set of questions regarding this subject was designed. To assess the face validity of the questionnaire, some professionals reviewed it. Finally, questions were distributed randomly in the questionnaire.

\subsection{Ethics Consideration}

This article has a license from the Research Ethics Committees of Shahid Sadoughi University of Medical Sciences, code IR.SSU.SPH.REC.1394.114.

\subsection{Statistical Analysis}

The analyses, including descriptive statistics (mean and standard deviation) and statistical tests ( $t$-test and ANOVA for the group comparison and Pearson's correlation for the relationship between variables), were performed using SPSS 16.

The researchers used the face validity, content validity, and construct validity to determine the validity of the questionnaire. In this study, face validity was examined using expert panel and necessary changes were made through simplification, meaning finding, deletion, or replacement of some words and questions.

In terms of content validity, the researchers requested five university professors in various fields to examine each item and give a score (essential $=3$, useful but not necessary $=2$, not necessary $=1$ ). Then, the validity of each question was determined using the Lawshe formula.

In addition, construct validity of the questionnaire was determined using the factor analysis method. For assessing the reliability and reproducibility of the questionnaire, the researchers used the Cronbach's alpha coefficient and test-retest method.

\section{Results}

Participants in this study were patients with cancer aged less than 18 years. The mean age of the patients was $9.54 \pm 3.97$ years and the mean duration of the cancer was $12.82 \pm 2.66$ months. The most common type of cancer among participants in this study was leukemia (57\%) and lymphoid malignancies (22\%).

In this study, face validity was examined using a panel of experts, including: physician, psychologist, health education specialist, pediatrician, and internist. They offered their suggestions for the appearance of the questionnaire, 
the use of proper words, and the use of proper grammar, as well as gave a score $(1=$ approve a question, $0=$ reject a question) to each questions. Based on their suggestions, the required changes were made in the questions and unnecessary questions were deleted. The results in terms of face validity indicated that the questions were verified by the expert panel in terms of clarity, relevance, and simplicity. According to statistics expert, a 4-point Likert-type scaling was used (never $=0$, sometimes $=1$, usually $=2$, always $=3$ ). In terms of content validity based on the Lawshe formula, all experts selected the necessary options for all questions. This indicated that the CVR (content validity ratio) for all questions equals one.

The results of the factor analysis are shown in Table 1. Based on the results of factor analysis using principal components analysis, three dimensions were extracted and confirmed. In this study, eigenvalues greater than one were considered. The factor loads greater than 0.3 were considered for selecting questions and placing them in the questionnaire. The load factor range was between 0.35 and 0.91 . Therefore, three dimensions that were identified and confirmed included: Daily activities and general problems (11 items), sleep problems (nine items), and mental states and emotions (five items). After performing a factor analysis, two questionnaires were removed from the questionnaire. Finally, the questionnaire included 25 questions.

According to the results, Cronbach's alpha coefficient for all dimensions of the questionnaire was more than 0.8 . ICC for the whole scale was 0.93 . Cronbach's alpha coefficients for each dimensions of the questionnaire are shown in Table 2. This indicates that the questionnaire has an appropriate reliability. According to Table 2, daily activities and general problems had the highest coefficients and sleep problems had the lowest one.

In addition, to determine the reliability of the questionnaire, researchers used test retest method. Test-retest is a method for assessing the reliability by administering the same test twice over a period of time. The questionnaire was completed by 40 people and repeated after 4 weeks on the same people. According to Table 2, ICC was more than 0.8 for all dimensions of the questionnaire. In addition, ICC was in the range of 0.84 to 0.92 . Furthermore, it was 0.92 for the whole scale. The ICC close to one shows the high reproducibility of the questionnaire.

In Table 3, the mean and standard deviation of the dimensions of cancer-related fatigue questionnaire has been shown. Based on Table 3, the mean of all dimensions, except the sleep problems, were higher than the possible range.

According to the results, by increasing the parents' education, the fatigue in children has been reduced. This relationship was statistically significant for mother's educa- tion. In this study, no significant differences were found between the gender and type of cancer with fatigue in the children.

\section{Discussion}

By considering the lack of questionnaire for measuring fatigue in children with cancer, the present study introduced a comprehensive instrument to examine fatigue in patients having cancer aged $4-18$ years. Fatigue is one of the important factors in the care of children with cancer. In this study, researchers tried to design a comprehensive tool to examine the fatigue in children with cancer based on the Iranian culture. This study showed that Introduced questionnaire is a valid and reliable instrument to collect information of children with cancer. In this study, professionals assessed the face validity of the questionnaire and offered their suggestions regarding the questionnaire's clarity and content. At this stage, three questions were removed and the rest of the questions were modified according to the expert panel.

One of the suitable methods for evaluating the construct validity, as the most important step in determining the validity of a questionnaire, is the use of factor analysis method. In this method, the questions in the questionnaire are categorized and construct validity is confirmed largely. In this study, the results of the factor analysis showed that the cancer-related fatigue questionnaire is a multidimensional questionnaire; which is on line with the results of the Kudubes et al., study for the Turkish population (8). These dimensions included: Daily activities and general problems, sleep problems, mental states, and emotions. In addition, the questionnaire used in Kudubes et al., study has three dimensions that included: General problems, sleep problems, and treatment problems (8). However, the questionnaire used in Kudubes et al. has 4 dimensions included: General problems, sleep problems, treatment problems, and cognitive problems (19). The Fatigue Scale-Child questionnaire assesses fatigue in children with cancer from the perspectives of the child, parents, and staff during the past week. This scale included 3 sub dimensions: Loss of energy altered sleeping patterns and mental changes (20).

Reliability is one of the most important features for studies designed to measure variables. Without a reliable tool, researchers cannot trust the results of the research and if the study is repeated, there is a significant difference with the previous phase. Although reliability is not a sufficient condition for trusting a questionnaire, it is a necessary condition (21). Cronbach's alpha is used to assess the reliability of the questionnaire in various sciences, including humanities and medical science (22). With the Cron- 


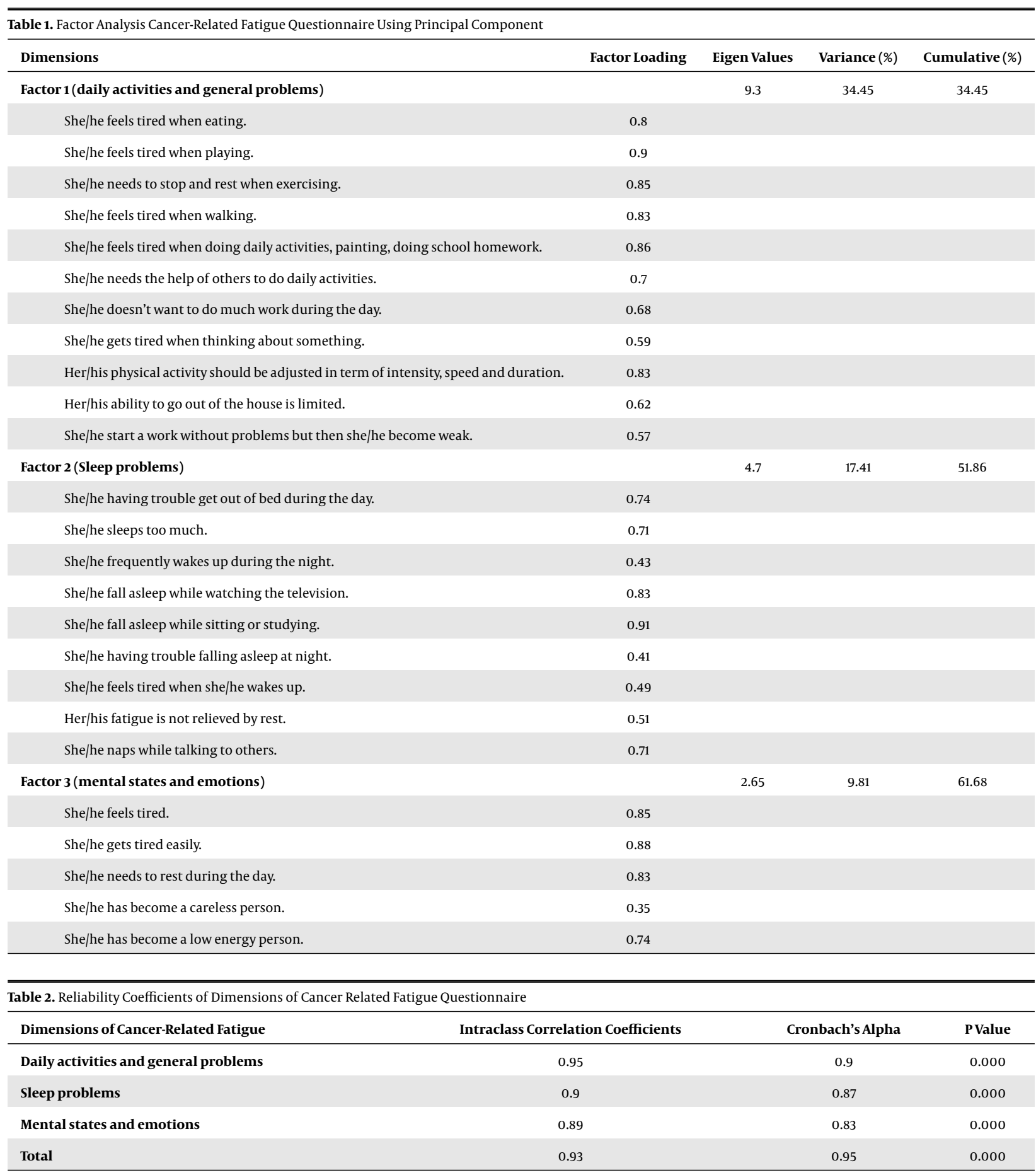

bach's alpha getting closer to one, the internal consistency between questions is greater, and as a result, questions will be more homogeneous. The results of this study indicated that the Cronbach's alpha coefficient for all dimensions of the questionnaire was higher than 0.8 and for the whole questionnaire was higher than 0.9. Therefore, questions of this questionnaire have an appropriate and acceptable correlation with each other. Cronbach's alpha for fatigue questionnaire in Kudubes et al., study was 0.98, which is consistent with the present study (8). 


\begin{tabular}{lcc}
\hline Table 3. Mean of Dimensions of Cancer-Related Fatigue Questionnaire & \\
\hline Dimensions of Cancer-Related Fatigue & Mean \pm SD & Range \\
\hline Daily activities and general problems & $25.66 \pm 0.76$ & $0-44$ \\
Sleep problems & $17.27 \pm 0.56$ & $0-36$ \\
Mental states and emotions & $12.9 \pm 0.33$ & $0-20$ \\
Total & $55.83 \pm 1.49$ & $0-100$ \\
\hline
\end{tabular}

Test-retest is a suitable method for determining the stability of the questionnaire at different times. In this method, the questionnaire is redistributed after a time period. Then, the correlation between two time periods is examined. ICC close to one indicated that the questionnaire has a high repeatability (22). Therefore, in this study, the questionnaire was completed by 40 people and it was repeated after four weeks on the same people. ICC was close to one for all dimensions of the questionnaire and whole questionnaire in this study. It supports the high stability of the questionnaire. If the questionnaire is used at different times, the same results will be obtained. It is consistent with the results of Kudubes et al., study for the Turkish population (8).

In this study, the mean fatigue score for patients that supports the high stability of the questionnaire was 52.42 \pm 1.91 . Considering that the score range for the fatigue is 0 -100 , the mean fatigue score in this study is moderate. It is consistent with the results of the Kudubes et al. studies (8, 19).

\subsection{Study Limitation}

The limitation of the present study is relying on parent's reports about children's fatigue.

\subsection{Conclusion}

This study showed that the validity and reliability of the cancer-related fatigue questionnaire are acceptable and suitable for Iranian culture, therefore, the present questionnaire can be used in other studies for examining fatigue in patients with cancer aged $4-18$ years in the Iranian population.

\section{Footnotes}

Authors' Contribution: Mahdieh Momayyezi and Dr. Mohammad Momayyezi conceptualized and designed the study, drafted the initial manuscript, designed the data collection instruments, and approved the final manuscript as submitted. Dr. Hossein Fallahzadeh carried out the initial analyses, reviewed and revised the manuscript, and approved the final manuscript as submitted. Ms. Fatemeh
Farzaneh and Soheila Bazm coordinated and supervised data collection, critically reviewed the manuscript, and approved the final manuscript as submitted.

Conflict of Interests: The authors declare that there is no conflict of interest.

Ethical Considerations: This article has a license from the Research Ethics Committees of Shahid Sadoughi University of Medical Sciences, code IR.SSU.SPH.REC.1394.64.

Financial Disclosure: The authors declared that this study received no financial support.

\section{References}

1. Etemadi A, Sadjadi A, Semnani S, Nouraie SM, Khademi H, Bahadori M. Cancer registry in Iran: A brief overview. Arch Iran Med. 2008;11(5):57780. [PubMed: 18759534]

2. Mousavi SM, Pourfeizi A, Dastgiri S. Childhood cancer in Iran.JPediatr Hematol Oncol. 2010;32(5):376-82. doi: 10.1097/MPH.ob013e3181e003f7. [PubMed: 20588194].

3. World Health Organization Regional Office for the Eastern Mediterranean. A strategy for cancer control in north Africa and the eastern Mediterranean region 2007. Eastern Mediterranean; 2010. Available from: http://www.emro.who.int/dsaf/dsa1002.pdf..

4. Kaatsch P, Steliarova-Foucher E, Crocetti E, Magnani C, Spix C, Zambon P. Time trends of cancer incidence in European children (1978-1997): Report from the automated childhood cancer information system project. Eur J Cancer. 2006;42(13):1961-71. doi: 10.1016/j.ejca.2006.05.014. [PubMed:16919764].

5. Hernandez BY, Green MD, Cassel KD, Pobutsky AM, Vu V, Wilkens LR. Preview of Hawaii cancer facts and figures 2010. Hawaii Med J. 2010;69(9):223-4. [PubMed: 20848386]. [PubMed Central: PMC3071205].

6. Ng SC, Ching JY, Chan V, Wong MC, Suen BY, Hirai HW, et al. Diagnostic accuracy of faecal immunochemical test for screening individuals with a family history of colorectal cancer. Aliment Pharmacol Ther. 2013;38(7):835-41. doi: 10.1111/apt.12446. [PubMed: 23957462].

7. Dupuis LL, Milne-Wren C, Cassidy M, Barrera M, Portwine C, Johnston DL, et al. Symptom assessment in children receiving cancer therapy: The parents' perspective. Support Care Cancer. 2010;18(3):281-99. doi: 10.1007/s00520-009-0651-1. [PubMed: 19513763].

8. Kudubes AA, Bektas M, Ugur O. Developing a scale for the assessment of fatigue in pediatric oncology patients aged 7-12 for children and parents. Asian Pac J Cancer Prev. 2014;15(23):10199-207. [PubMed: 25556448].

9. Minton O, Strasser F, Radbruch L, Stone P. Identification of factors associated with fatigue in advanced cancer: A subset analysis of the European palliative care research collaborative computerized symptom assessment data set. J Pain Symptom Manage. 2012;43(2):226-35. doi: 10.1016/j.jpainsymman.2011.03.025. [PubMed: 21839608].

10. Holliday EB, Dieckmann NF, McDonald TL, Hung AY, Thomas CR, Jr, Wood LJ. Relationship between fatigue, sleep quality and inflammatory cytokines during external beam radiation therapy for prostate cancer: A prospective study. Radiother Oncol. 2016;118(1):105-11. doi: 10.1016/j.radonc.2015.12.015. [PubMed: 26743832]. [PubMed Central: PMC5863280].

11. Nishiura M, Tamura A, Nagai H, Matsushima E. Assessment of sleep disturbance in lung cancer patients: Relationship between sleep disturbance and pain, fatigue, quality of life, and psychological distress. Palliat Support Care. 2015;13(3):575-81. doi: 10.1017/S1478951513001119. [PubMed: 24524428]. 
12. Berger AM, Abernethy AP, Atkinson A, Barsevick AM, Breitbart WS, Cella D, et al. NCCN clinical practice guidelines cancer-related fatigue. J Natl Compr Canc Netw. 2010;8(8):904-31. [PubMed: 20870636].

13. Gibson F, Garnett M, Richardson A, Edwards J, Sepion B. Heavy to carry: A survey of parents' and healthcare professionals' perceptions of cancer-related fatigue in children and young people. Cancer Nurs. 2005;28(1):27-35. [PubMed: 15681979].

14. Jalmsell L, Kreicbergs U, Onelov E, Steineck G, Henter JI. Symptoms affecting children with malignancies during the last month of life: A nationwide follow-up. Pediatrics. 2006;117(4):1314-20. doi: 10.1542/peds.2005-1479. [PubMed: 16585329].

15. Momayyezi M, Fallahzadeh H, Farzaneh FF, Momayyezi M. Sleep quality and disturbances in children and adolescents with cancers: A cross-sectional study. Int J Pediat. 2018;6(4):7529-42.

16. Lai JS, Haertling T, Weinstein J, Rademaker AW, Goldman S. A crosssectional study of carnitine deficiency and fatigue in pediatric cancer patients. Childs Nerv Syst. 2016;32(3):475-83. doi: 10.1007/s00381-0152983-0. [PubMed: 26812488]. [PubMed Central: PMC5872812].

17. Cruciani RA, Dvorkin E, Homel P, Malamud S, Culliney B, Lapin J, et al. Safety, tolerability and symptom outcomes associated with l-carnitine supplementation in patients with cancer, fatigue, and carnitine deficiency: A phase i/ii study. J Pain Symptom Manage. 2006;32(6):551-9. doi: 10.1016/j.jpainsymman.2006.09.001. [PubMed: 17157757].

18. Momayyezi M, Fallahzadeh H, Momayyezi M. Construction and validation the lifestyle questionnaire related to cancer. Iran J Cancer Prev. 2015;8(5). e3965. doi: 10.17795/ijcp-3965. [PubMed: 26634112]. [PubMed Central: PMC4667238].

19. Bektas M, Kudubes AA. Developing scales for the assessment of fatigue in Turkish pediatric oncology patients aged 13 - 18 and their parents. Asian Pac J Cancer Prev. 2014;15(22):9891-8. [PubMed: 25520124].

20. Hockenberry MJ, Hinds PS, Barrera P, Bryant R, Adams-McNeill J, Hooke $\mathrm{C}$, et al. Three instruments to assess fatigue in children with cancer: The child, parent and staff perspectives. J Pain Symptom Manage. 2003;25(4):319-28. [PubMed: 12691683].

21. Ghasemi V. Cronbach coefficient alpha and some of its specialties with emphasis on applications in social research. Res Bull Isfahan Univ (Hum). 2005;19(2):155-74.

22. Rahaei Z, Ghofranipour F, Morowatisharifabad MA. [Psychometric properties of a protection motivation theory questionnaire used for cancer early detection]. J School Public Heal Inst Public Heal Res. 2015;12(3):69-79. Persian. 